[3] https://www.das-score.nl/das28/en/difference-between-the-das-and-das28/ importance-of-das28-and-tight-control/eular-response-criteria.html

Acknowledgement: This study was supported by the Bundesministerium für Bildung und Forschung (BMBF) project "ArthroMark", subproject no. 7.

Disclosure of Interests: Sarah Ohrndorf: None declared, Lisa Ridha : None declared, Anne-Marie Glimm: None declared, Gerd Rüdiger Burmester Consultant for: Roche, Sanofi-Genzyme, Speakers bureau: Roche, Sanofi-Genzyme, Gabriela Schmittat: None declared, Marina Backhaus: None declared, Jens Klotsche: None declared DOI: 10.1136/annrheumdis-2019-eular.6694

\section{AB1166 SUBCLINICAL ENTHESEAL INVOLVEMENT IN LOWER LIMBS IN PATIENTS WITH TYPE 2 DIABETES MELLITUS: AN ULTRASOUND STUDY}

Mohamed Omar ${ }^{1}$, Mohamed Abdelkareem², Mohammed Moneer ${ }^{2}$, Mohamed Elwan'. ' 1 Alazher, Rheumatology, Asuit, Egypt, ${ }^{2}$ Alazher, Rheumatology, Assuit, Egypt

Background: Diabetes mellitus (DM) is a group of metabolic diseases characterized by hyperglycemia, which results from defects in insulin secretion, insulin action, or both (1).

DM impacts the connective tissue and causes various changes in periarticular and articular structures.An increase in enthesopathic complications in diabetic patients such as patellar tendinitis bursitis, achilles tendinopathy and plantar fasciitis, was observed In many studies.

Objectives: This study aims to detect subclinical lower limb enthesitis in type 2 DM by musculoskeletal ultrasonography \& its relation to poor glycemic control \& disease duration

Methods: This study was carried out on 80 persons. Diabatic group forty patients diagnosed as diabetes according to ADA diagnostic criteria. Control group Forty apparent healthy volunteers both groups was matched at age and sex.Sonographic evaluations and scoring were performed according to Glasgow Ultrasound Enthesitis Scoring System(GUESS) on the enthses of both lower limbs.

Results: At the diabetic group the Musculoskeletal Ultrasound findings was as following. The Quariceps tendon enthesitis in 30 patients (75\%), proximal patellar enthesitis was in 28 patients $(70 \%)$, Distal patellar enthesitis in 22 patients (55\%), Achilles enthesitis in 27 patients (67.5) \& planter aponeurosis enthesitis in 25 patients $(55 \%)$. There is a statistically significant difference between age and Ultrasound findings $p$. value

$<0.05$. There is a statistically significant correlation between disease duration and (proximal patellar ligament,Achilles tendon, quadriceps tendon and plantar fascia) thickness p. value $<0.05$.

Conclusion: Entheseal abnormalities can be documented by ultrasonography in clinically asymptomatic patients with Diabetes. These findings could be related to a subclinical entheseal inflammation.

\section{REFERENCES}

[1] Okur SC, YaseminPekinDogan, Murat Mert, OzgeAksu, OzerBurnaz, Nil SayinerCaglar(2017): Ultrasonographic Evaluation of Lower Extremity Entheseal Sites in Diabetic Patients Using Glasgow Ultrasound Enthesitis Scoring System Score, Journal of Medical Ultrasound (2017), http://dx.doi. org/10.1016/j.jmu..03.011

[2] SMITH, L., BURNET, S. \& MCNEIL, J. (2003): Musculoskeletal manifestations of diabetes mellitus. British journal of sports medicine, 37, 30-35.

Disclosure of Interests: None declared

DOI: 10.1136/annrheumdis-2019-eular.3468

\section{AB1167 RELIABILITY AND VALIDITY OF ULTRASOUND PATHOLOGIES IN KNEE OSTEOARTHRITIS FOR SEMI- QUANTITATIVE AND QUANTITATIVE METHODS WITH MRI AS A REFERENCE}

Win Min Oo ${ }^{1}$, James Linklater ${ }^{2}$, Xia Wang ${ }^{1}$, Matthew Daniel ${ }^{1}$, Danielle Pryke ${ }^{2}$, Shirley $\mathrm{Yu}^{1}$, Leticia Deveza ${ }^{1}$, Vicky Duong ${ }^{1}$, David Hunter ${ }^{1} .{ }^{1}$ Rheumatology Department, Royal North Shore Hospital and Institute of Bone and Joint Research, Kolling Institute, University of Sydney, Australia, Sydney, Australia; ${ }^{2}$ Department of Musculoskeletal Imaging, Castlereagh Sports Imaging Center, St. Leonards, Sydney, Australia, Sydney, Australia

Background: Ultrasound can visualize both structural and inflammatory changes of osteoarthritis (OA) with greater accessibility. Therefore, the Outcome Measures in Rheumatology (OMERACT) Ultrasound Task Force recently proposed a semi-quantitative knee ultrasound scoring system ${ }^{1}$ which requires further validation.

Objectives: To investigate inter-rater reliability (IRR) of ultrasound pathologies between three raters with varying experiences and examine construct validity of these features against magnetic resonance imaging (MRI) using OMERACT semi-quantitative ultrasound image atlas and quantitative ultrasound measures.

Methods: According to standardized OMERACT scanning protocol, 20 participants with symptomatic and radiographic knee OA were scanned dynamically with a multi-frequency linear transducer $(6-18 \mathrm{MHz})$ of Aplio Platinum 500 machine, Toshiba, Japan.

Following an initial calibration of ultrasound scores using the image atlas, three raters with varying experience (a physician operator certified with RhMSUS, a musculoskeletal ultrasonographer and a medical student) independently obtained semi-quantitative scores (0-3) for synovitis and power Doppler activity in suprapatellar recess, medial osteophytes and medial meniscal extrusion, as well as quantitative measurements (mm) of synovitis, effusion, synovial hypertrophy, medial osteophyte and meniscal extrusion (maximal measures)

Semi-quantitative MRI Osteoarthritis Knee Score (MOAKS) scores of effusion-synovitis, osteophyte and meniscal extrusion were independently calculated by an experienced researcher. 3-T Sagittal proton-density (PD) weighted fat-suppressed turbo spin-echo (TSE) non-contrast MRI sequences were used for quantitative effusion-synovitis, and coronal plane for osteophyte and meniscal extrusion.

Weighted kappa coefficient $(\mathrm{Kw})$ or intra-class correlation coefficient (ICC) were calculated for IRR, Spearman's rank ( $r s$ ) and Pearson (rp) correlation coefficients for construct validity and Bland-Altman plots for extent of agreement between ultrasound and MRI.

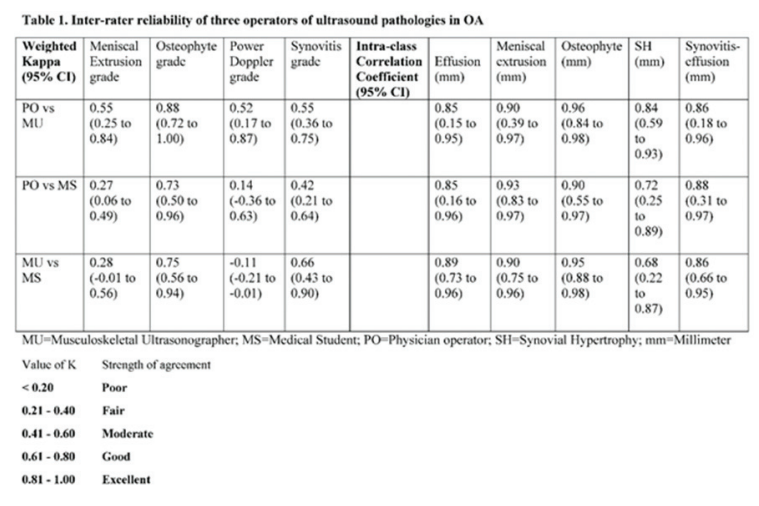

Figure 1

\begin{tabular}{|c|c|c|c|c|c|c|}
\hline $\begin{array}{l}\text { Ultrasound vs } \\
\text { MRI }\end{array}$ & Synovitis grade & $\begin{array}{l}\text { Meniscal } \\
\text { extrusion Grade }\end{array}$ & Osteophyte Grade & $\begin{array}{l}\text { Synovitis } \\
(\mathrm{mm})\end{array}$ & $\begin{array}{l}\text { Meniscal } \\
\text { extrusion (mm) }\end{array}$ & $\begin{array}{l}\text { Osteophyte } \\
\text { (mm) }\end{array}$ \\
\hline Spearman (rs) & $\begin{array}{l}0.48 \\
P=0.03\end{array}$ & $\begin{array}{l}0.27 \\
P=0.26\end{array}$ & $\begin{array}{l}0.36 \\
\mathrm{P}=0.12\end{array}$ & & & \\
\hline Pcarson (rp) & & & & $\begin{array}{l}0.51 \\
\mathrm{P}=0.02\end{array}$ & $\begin{array}{l}0.67 \\
P=.001\end{array}$ & $\begin{array}{l}0.54 \\
P=.02\end{array}$ \\
\hline
\end{tabular}

Figure 1

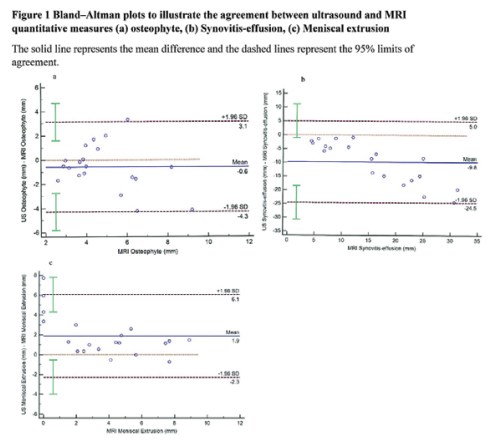

Figure 1 
Results: Demographics were $60.2 \pm 8.3$ years of age, $65 \%$ female, BMl of $28.6 \pm 4.5$ of $\mathrm{kg} / \mathrm{m} 2 ; 60 \%$ with Kellgren and Lawrence $(\mathrm{KL})$ grade 2 and $40 \%$ with a $\mathrm{KL}$ grade 3 . Semi-quantitative OMERACT ultrasound scores (Table.1) revealed good to excellent IRR ( $\mathrm{Kw}=0.73-0.88)$ for osteophyte, and moderate to good IRR $(\mathrm{Kw}=0.42-0.66)$ for synovitis. Conversely, quantitative measures of ultrasound pathologies had excellent IRR (ICC= 0.84-0.95) except for synovial hypertrophy (ICC=0.67-0.72).

A significant association was found between semi-quantitative ultrasound synovitis and $\mathrm{MRI}$ effusion-synovitis ( $\mathrm{rs}=0.48, \mathrm{p}=0.03$ ). All three quantitative ultrasound measurements were significantly associated with quantitative scores of MRI (Table. 2).

In Bland-Altman plots for quantitative measurements, there were systemic offsets of $0.6 \mathrm{~mm}, 1.9 \mathrm{~mm}$ and $9.8 \mathrm{~mm}$ for osteophyte and meniscal extrusion and synovitis respectively (Fig. 1)

Conclusion: These is moderate to good IRR between operators with varying experience using the OMERACT knee scoring image atlas for osteophyte and synovitis. While quantitative ultrasound measurements showed excellent IRR and significant association with MRI quantitative outcomes, the absolute feature-specific agreement is called into question.

\section{REFERENCES}

[1] Bruyn, G.A.W., et al., An OMERACT reliability exercise of inflammatory and structural abnormalities in patients with knee osteoarthritis using ultrasound assessment. Annals of the Rheumatic Diseases, 2016. 75(5): p. 842-846.

Disclosure of Interests: None declared

DOI: 10.1136/annrheumdis-2019-eular.2134

\section{AB1168 $\quad$ SIGLEC1/CD169 IS A SENSITIVE MARKER FOR MONOGENIC INTERFERONOPATHIES}

Banu Orak $^{1,2}$, Marc Nikolaus ${ }^{1,3}$, Ellen Knierim ${ }^{1,3}$, Angela Kaind $^{1,3}$ Manuela Theophil ${ }^{4}$, Axel Panzer ${ }^{4}$, Barbara Zieba ${ }^{5}$, Frédéric Ebstein ${ }^{5}$, Elke Krüger ${ }^{5}$, Nadine Unterwalder ${ }^{6}$, Christian Meisel ${ }^{6}$, Tilmann Kallinich ${ }^{1,2} .{ }^{1}$ Berlin, Center for Chronically Sick Children (Sozialpädiatrisches Zentrum, SPZ) - Charité Universitätsmedizin Berlin, Berlin, Germany; ${ }^{2}$ Berlin, Department of Pediatrics, Division of Pneumonology and Immunology with intensive Medicine, Charité Universitätsmedizin Berlin, Berlin, Germany; ${ }^{3}$ Berlin, Department of Pediatric Neurology, Charité - Universitätsmedizin Berlin, Berlin, Germany; ${ }^{4}$ Berlin, Pediatric Neurology, DRK Klinikum Berlin-Westend, Berlin, Germany, ${ }^{5}$ Greifswald, Institute of Medical Biochemistry and Molecular Biology, Universitätsmedizin Greifswald, Greifswald, Germany, ${ }^{6}$ Berlin, Department of Immunology, Labor Berlin GmbH, Berlin, Germany

Background: Monogenic Interferonopathies are a rare group of inflammatory diseases that are difficult to diagnose in the onset phase given the lack of well-defined disease-markers. ${ }^{1} \mathrm{~A}$ correlation with interferon-stimulated genes (ISG) has been reported for SIGLEC1 (syn. CD169), in systemic lupus erythematodes (SLE). ${ }^{2}$ Furthermore, expression of SIGLEC1 on monocytes is the second highest ISG in SLE. ${ }^{3}$

Objectives: To study the relevance of SIGLEC1 as a putative diagnostic marker for early detection of interferonopathies.

Methods: Clinical data, classical inflammatory markers, blood count values and genetic information were obtained from the medical files of eight patients with genetically confirmed monogenic interferonopathies. SIGLEC1 expression was measured by flow cytometry with a highly standardized quantitative assay with a reference range in healthy controls less than 2500 SIGLEC1 molecules/monocyte. Additionally, transcriptional level of SIGLEC1, IFI44L, IFI27, ISG15 and RSAD2 as type I Interferon stimulated genes were assessed by real-time PCR

Results: All eight patients with interferonopathies carried mutations in the genes TREX-1 $(\mathrm{n}=3)$, IFIH-1 $(\mathrm{n}=2)$, SAMDH1 $(\mathrm{n}=2)$ and RNASE2HB $(n=1)$. Mean age of patients was 12 years (range 6 months to 49 years). Six of eight patients showed neurological symptoms consistent with Aicardi-Goutieres-Syndrome presenting developmental delay and microcephaly. Five patients showed abnormalities on cranial MRI including periventricular calcifications and corpus callosum thinning. Two patients were diagnosed with Singleton-Merten-Syndrome presenting abnormal ossification of extremities and dental anomalies. One patient with homozygous TREX1 mutation presented with postnatal glaucoma, microcephaly, sensorimotor polyneuropathia and recurrent fever with persistent chilblain lesions. All patients had elevated SIGLEC1 levels (mean molecules/monocyte +/ SD: $10272+/-3746$ ) without high levels of standard inflammatory markers. In six patients, elevated SIGLEC1 expression showed dysregulation of the type 1 interferon pathway prior to genetic testing. The relative expression $(\Delta \mathrm{Ct})$ of all ISG's was significantly elevated in comparison to healthy controls (SIGLEC1 $\mathrm{p}=0,0167$, ISG15 $\mathrm{p}=0,0015$, RSAD2 $\mathrm{p}=0,0067$, IFIL44 $\mathrm{p}=0,0001$, IFI27 $\mathrm{p}=0,0056)$.

Conclusion: We report high expression of SIGLEC1 in monogenic interferonopathies like Aicardi-Goutières-Syndrome.

Therefore, SIGLEC1 qualifies as an easy accessible and cheap diagnostic marker to screen patients with suspected interferonopathy.

\section{REFERENCES}

[1] Crow, Y.J., Type I interferonopathies: mendelian type I interferon up-regulation. Curr Opin Immunol, 2015. 32: p. 7-12.

[2] Rose, T., et al., Are interferon-related biomarkers advantageous for monitoring disease activity in systemic lupus erythematosus? A longitudinal benchmark study. Rheumatology (Oxford), 2017. 56(9): p. 1618-1626.

[3] Biesen, R., et al., Sialic acid-binding lg-like lectin 1 expression in inflammatory and resident monocytes is a potential biomarker for monitoring disease activity and success of therapy in systemic lupus erythematosus. Arthritis Rheum, 2008. 58(4): p. 1136-45.

Disclosure of Interests: Banu Orak: None declared, Marc Nikolaus: None declared, Ellen Knierim: None declared, Angela Kaindl: None declared, Manuela Theophil: None declared, Axel Panzer: None declared, Barbara Zieba: None declared, Frédéric Ebstein: None declared, Elke Krüger: None declared, Nadine Unterwalder: None declared, Christian Meisel: None declared, Tilmann Kallinich Grant/research support from: Novartis, Speakers bureau: Sobi, Roche, Novartis, CLB DOI: 10.1136/annrheumdis-2019-eular.5506

\section{AB1169 ASSOCIATION OF ANXIETY, DEPRESSION WITH ULTRASONOGRAPHIC EVALUATION OF THE JOINTS, DISEASE ACTIVITY, FUNCTIONAL DISABILITY AND QUALITY OF LIFE IN PATIENTS WITH RHEUMATIC DISEASES:}

Rita Osipyants $^{1}$, Victoria Nadtocheeva ${ }^{2}$, Madina Bogdanova ${ }^{3}$, Marina Kanevskaya ${ }^{4}$, Temuri Mirilashvili ${ }^{5} .{ }^{1}$ Department of Internal Diseases, RUDN University; Eramishantsev City Clinical Hospital, Moscow, Russian Federation; '2Department of Internal Diseases, RUDN University, Moscow, Russian Federation; ${ }^{3} \mathrm{FSBI}$ "Polyclinic №1" of the Administrative Department of the President of the Russian Federation, Moscow, Russian Federation; ${ }^{4}$ Sechenov Moscow Medical University, Moscow, Russian Federation; ${ }^{5}$ Eramishantsev City Clinical Hospital, Moscow, Russian Federation

Objectives: The aim of this study is to evaluate anxiety and depression disorders and to study their correlation with disease activity, ultrasonographic (US) finding indicative of synovitis, functional status and quality of life in patients (pts) with rheumatic diseases.

Methods: 39 pts (F/M-29/10) with rheumatoid arthritis (RA, $n=17)$, ankylosing spondylitis (AS, $n=10)$, psoriatic arthritis (PsA, $n=12$ ) were included. All RA pts fulfilled the ACR/EULAR 2010, AS pts - the ASAS, PsA pts the CASPAR criteria. 20 pts (8 RA, 6 AS, 6 PsA) were performed US examinations (Mindray DC-N6 (China), C5-2, L10-13 MHz probes) included bilateral of the hip and knee joints. Each joint was scored according to the OMERACT definitions of pathology. Functional status (BASFI), disease activity indices (DAS28-CRP, ASDAS-CRP, DAPSA), anxiety and depression levels (Hads-A, Hads-D) and quality of life (RAPID3, EQ-5D) were assessed. The Mann-Whitney U-test was applied for intergroup comparison and correlation was evaluated using a Spearman's Rank two-tailed test ( $R$ value is shown).

Results: Median age of 39 pts - $52(44 ; 61)$ years and the body mass index - 28 (23;30). Mean anxiety index was $6.7 \pm 3.4$, depression index 6.6 \pm 3.3 . Anxiety disorders were detected in $13 / 39$ pts $(33.3 \%)$, depression disorders in $17 / 39$ pts $(43.5 \%)$. Pts with Hads-D $\geq 8 \quad(n=17)$ had higher score ASDAS-CRP $(4.6(4.2 ; 5.0)$ vs 3.1 (2.7;3.9), $\mathrm{p}<0.01)$, BASFI $(7.2$ $(6 ; 7.3)$ vs $4.2(3 ; 5.5), \quad \mathrm{p}<0.05)$, EQ-5D index $(9.8 \pm 1.2$ vs $8.5 \pm 1.6$, $\mathrm{p}<0.01)$ and the same tendency to US score and DAS28-CRP compared pts without depression. But pts with Hads-A $>8 \quad(n=13)$ had only higher EQ-5D $(10 \pm 1.2$ vs $8.7 \pm 1.6, \quad \mathrm{p}<0.005)$ and RAPID3 $(17.1 \pm 5.8$ vs 15.6 $\pm 1.6, \mathrm{p}<0.05)$. However, US - synovitis of the hip joints was positively correlated with Hads-D $(r=0.713, p<0.05)$ and Hads-A $(r=0.587, p<0.05)$. Conclusion: Anxiety and depression more common in patients with active rheumatic diseases. Psychological disorders are associated with disease activity, US- finding of synovitis and the severity of functional disability. Disclosure of Interests: None declared DOI: 10.1136/annrheumdis-2019-eular.8344 